REGARDS

SUR L'ECONOMIE ALLEMANDE

BULLETIN ECONOMIQUE DU CIRAC

\section{Regards sur l'économie allemande}

Bulletin économique du CIRAC

$92 \mid 2009$

Varia

\title{
Accords de distribution et européanisation du droit allemand de la concurrence
}

Géraldine Demme

\section{(2) OpenEdition}

\section{Journals}

Édition électronique

URL : http://journals.openedition.org/rea/3758

DOI : 10.4000/rea.3758

ISBN : 978-2-8218-0880-5

ISSN : 1965-0787

Éditeur

CIRAC

Édition imprimée

Date de publication : 1 juillet 2009

Pagination : 27-34

ISSN : 1156-8992

Référence électronique

Géraldine Demme, "Accords de distribution et européanisation du droit allemand de la concurrence ", Regards sur l'économie allemande [En ligne], 92 | juillet 2009, mis en ligne le 01 juillet 2011, consulté le 01 mai 2019. URL : http://journals.openedition.org/rea/3758 ; DOI : 10.4000/rea.3758 


\section{Accords de distribution et européanisation du droit allemand de la concurrence}

\section{Géraldine Demme}

Un fabricant peut-il interdire aux détaillants d'utiliser le portail Internet eBay pour la vente de ses produits ? Le tribunal régional de Berlin a condamné clairement une telle pratique dans un arrêt du 21 avril 2009 (Az. 16 O 729/07). Contrairement à une décision de son homologue de Mannheim en 2008 dans une affaire tout à fait similaire (Az. 7 O 263/07), le tribunal berlinois a rejeté l'argument du fabricant Scout - une marque réputée de cartables pour écoliers en Allemagne que la vente sur eBay nuirait à l'image de sa marque. Le fabricant ayant fait appel de cette décision, c'est le tribunal régional supérieur de Berlin qui sera chargé de se prononcer de nouveau sur cette question.

Si les réflexions sur le commerce électronique sont d'une brûlante actualité, tant à l'échelle nationale que communautaire, avec la mise en ligne le 26 mai 2009 du rapport sur les opportunités et les freins du commerce électronique par la Commission européenne, la question que pose cette affaire va bien au-delà du domaine spécifique de l'e-commerce : dans quelle mesure un fabricant peut-il limiter la liberté commerciale des revendeurs dans la distribution de ses produits?

L'Allemagne s'est montrée pendant longtemps très libérale, en remettant à la libre négociation entre fabricant et distributeur le soin de régler la question de la mesure des limitations que le premier pouvait apporter à la latitude du second dans la commercialisation des produits. Les juristes ainsi que les milieux économiques concernés étaient intimement persuadés que cette solution représentait un progrès par rapport au système européen plus strict. Le changement survenu en Allemagne avec l'abandon de cette solution ne s'explique ainsi que par la volonté d'éviter au maximum toute discordance entre droits allemand et communautaire. La convergence des droits qui en résulte est bénéfique, tant pour les entreprises allemandes souhaitant intégrer le marché commun que pour les autorités et tribunaux en charge des affaires nationales et européennes.

\section{La distribution, un des secteurs les plus dynamiques du marché allemand}

La " distribution », selon une définition courante, constitue l'action économique d'intermédiation entre la production matérielle et la consommation. L'activité de distribution a pour fonction d'assurer de manière optimale la disponibilité des produits ou des services aux consommateurs dans les lieux, temps et conditions les meilleurs. Par sa fonction, le secteur de la distribution agit sur la vie quotidienne de chaque individu; par son importance, il exerce une influence déterminante sur la vie économique et sociale en Allemagne.

Le développement des marchés de biens et services a conduit à une intensification de la concurrence entre participants. C'est ce déploiement des rapports concurrentiels et le mouvement vers la spécialisation qui a favorisé la complexification des relations économiques liées à la distribution entre agents économiquement trop faibles pour disposer d'une organisation verticale intégrée. Cette dernière implique qu'une entreprise dispose de suffisamment de moyens et d'infra-

La spécificité de la distribution indépendante 
L'antinomie entre l'indépendance juridique des entreprises ...

.... et l'interdépendance économique de leurs actions

Nuance fondamentale : les ententes verticales ne sont pas forcément anticoncurrentielles structures pour assurer l'ensemble des étapes du processus économique, de la fabrication à la mise à disposition du produit au consommateur final. Remède spontané aux déséquilibres structurels entre entreprises concurrentes de dimensions différentes, la distribution indépendante s'est répandue à tous les milieux économiques, aussi divers soient-ils: la vente d'automobiles, la restauration rapide, les circuits de produits de luxe, les chaînes de coiffure, n'en constituent que des exemples parmi d'autres. Elle implique la participation successive de plusieurs entreprises autonomes au même processus économique, allant de la production à la distribution. La franchise en constitue l'exemple le plus connu dans la vie quotidienne des consommateurs.

Si le fabricant peut aisément contrôler la détermination et l'application effective des prix et conditions de vente aux consommateurs dans l'hypothèse d'une distribution intégrée - la chaîne de décision étant exclusivement interne à une même entreprise -, de telles facultés lui sont en principe fermées lorsque son activité repose sur un système de distribution indépendante. En effet, le droit de la concurrence prohibe la coordination entre entreprises autonomes, susceptible de restreindre la concurrence. Peuvent ainsi constituer des restrictions de concurrence, par exemple les accords conduisant à la répartition de zones de revente, les conventions délimitant les sources d'approvisionnement, ou encore les ententes procédant à la fixation en commun du prix final au consommateur. En effet, partant de l'idée que le maintien du plus grand nombre d'entreprises autonomes serait le moyen le plus favorable à la situation concurrentielle sur le marché, certains considèrent qu'il y a restriction de concurrence dès lors qu'une clause restreint la liberté d'action commerciale d'une des entreprises, quel que soit l'élément de la liberté de décision concerné et quel que soit le but recherché par les parties de l'accord. Ils raisonnent alors dans le cadre du modèle de la concurrence pure et parfaite. II s'agit ici d'entendre de la manière la plus littérale le principe fondamental de la liberté d'entreprendre.

Juridiquement indépendants, les différents intervenants d'un même système de distribution sont toutefois liés très étroitement sur le plan économique du fait de leur communauté d'intérêt au succès d'un même produit ou service auprès des consommateurs. Ce contexte confère toute sa spécificité, voire sa dimension paradoxale, aux accords de distribution indépendante. En effet, pour augmenter l'efficacité du réseau dans son ensemble, il est primordial que les différents opérateurs puissent coordonner un minimum leurs actions. La réussite de la structure repose pour une grande partie sur la cohérence de la méthode commerciale adoptée. Ce n'est qu'autant que la distribution indépendante fédère et coordonne qu'elle favorise l'introduction et la persistance d'une structure verticale à même de se poser en nouvelle force concurrentielle sur le marché.

Ainsi, loin d'être toujours nocive à la concurrence, la coordination entre entreprises intervenant à différents stades du marché (on parle alors d' " entente verticale ») a un caractère ambivalent : souvent défavorable à la concurrence intramarque (ou intra-brand, c'est-à-dire la concurrence qui se produit à l'intérieur même de la structure verticale), elle est en principe bénéfique à la concurrence inter-marques (ou inter-brand, c'est-à-dire la concurrence entre les différentes structures verticales présentes sur le même marché). Dans certains cas, de tels accords constituent une restriction de concurrence, mais elles sont plus souvent source de gains d'efficience économique en augmentant la compétitivité du réseau dans son ensemble. Les ententes verticales ne peuvent donc pas être considérées a priori comme suspectes ou favorables à la concurrence. Signe de la force de ce constat, grand nombre de pays, ainsi que l'Union européenne, ont été amenés à reconsidérer leur approche juridique des ententes verticales fondée sur une qualification trop formelle et abstraite conduisant à une interdiction de principe en cas de limitation de l'autonomie commerciale d'une entreprise. Ils lui préfèrent une appréciation plus concrète sur la base des effets économiques réels de l'accord sur le marché. Le droit allemand a suivi toutefois un cheminement très spécifique en la matière. 


\section{L'évolution du droit allemand des ententes}

La loi allemande contre les entraves à la concurrence (Gesetz gegen Wettbewerbsbeschränkungen, GWB) distinguait traditionnellement les restrictions horizontales (qui existent entre entreprises concurrentes, comme c'est le cas d'un cartel de prix entre différents fabricants), des restrictions verticales (qui ont lieu entre opérateurs intervenant à différents stades du marché, comme c'est le cas dans les accords de distribution entre fabricant et distributeur). Seules les premières étaient sujettes à l'interdiction du $\S 1 \mathrm{du}$ GWB. Les ententes verticales mises à part celles relatives à l'imposition du prix de revente - étaient soumises à un simple contrôle de l'abus (Missbrauchskontrolle) qui, selon l'ancien $\S 16$ du GWB, n'ouvrait qu'une possibilité d'intervention limitée aux autorités de concurrence (Kartellbehörden). En revanche, le système communautaire adopte une approche plus générale des rapports entre entreprises indépendantes et a prévu dès son origine dans l'article $81, \S 1$ du Traité CE une interdiction de principe des ententes restrictives, tant horizontales que verticales. Cette interdiction est toutefois assortie d'un système d'exemption : la prohibition ne s'applique pas si les avantages sociaux et économiques dépassent les effets anticoncurrentiels de l'accord.

L'entente échappe ainsi à la prohibition de l'article $81, \S 1$ du Traité CE si elle satisfait aux quatre conditions cumulatives énumérées à l'article $81, \S 3$ du Traité CE, à savoir : (1) générer des gains d'efficacité économique dont (2) une partie équitable est répercutée aux utilisateurs ; (3) être indispensable à la réalisation de ces gains d'efficacité et (4) ne pas éliminer la concurrence, ni totalement, ni pour une partie substantielle des produits en cause.

Les accords qui remplissent ces conditions sont alors soustraits à l'interdiction. Plutôt que de vérifier au cas par cas si les ententes satisfont à ces critères, la Commission peut arrêter des règlements d'exemption par catégorie d'accords. Les accords contenus dans ces règlements sont alors présumés, jusqu'à preuve du contraire, satisfaire aux quatre conditions susmentionnées.

Si la $6^{\mathrm{e}}$ réforme du GWB en 1998 a eu pour objectif d'harmoniser le droit allemand avec le droit communautaire, une transposition de l'interdiction générale qui inclurait alors les ententes verticales a été fermement écartée. Les juristes allemands, ainsi que les milieux économiques concernés, étaient persuadés que le système de contrôle de l'abus représentait un progrès par rapport au principe d'interdiction générale du droit communautaire. Bien que cette appréciation reste inchangée en Allemagne, des considérations d'ordre pratique liées à l'adoption du Règlement communautaire $n^{\circ} 1 / 2003$ ont finalement amené le législateur allemand à consacrer le principe d'interdiction générale : les autorités et juridictions nationales sont désormais obligées de mettre en œuvre le droit communautaire dans tous les cas d'affectation du commerce entre États membres et ne peuvent appliquer parallèlement qu'un droit national convergent. Étant donné l'interprétation large par la Commission européenne du critère d'affectation du commerce entre États membres, la plupart des situations seront désormais soumises à l'application du droit communautaire. Or, le maintien de règles nationales divergentes pour quelques cas purement internes va à l'encontre d'une allocation optimale des ressources juridiques. A l'inverse, la convergence entre droits allemand et communautaire évite aux tribunaux des erreurs de jugements ou des contradictions liées à la délimitation incertaine du champ d'application des règles nationales ou européennes.

Sans obliger les droits nationaux à s'aligner sur sa solution, c'est toutefois le droit communautaire qui a donné l'impulsion pour la $7^{\mathrm{e}}$ réforme du GWB en Allemagne. Cette réforme, entrée en vigueur le $1^{\text {er }}$ juillet 2005 , a réalisé une harmonisation complète du droit allemand avec le droit communautaire : la distinction entre restrictions verticales et horizontales a été supprimée, les deux types de restrictions étant désormais soumis à l'interdiction du §1 du GWB: "Tous accords entre entreprises, toutes décisions d'associations d'entreprises et toutes
Droit allemand traditionnel : une conception plus souple que celle du droit communautaire

L'allocation optimale des ressources judiciaires, ...

... raison principale de l'alignement du droit allemand sur le droit communautaire 
Un alignement rendu possible grâce à la modernisation préalable du droit communautaire

Abandon partiel de souveraineté du législateur allemand au bénéfice du législateur communautaire pratiques concertées qui ont pour objet ou pour effet d'empêcher, de restreindre ou de fausser le jeu de la concurrence, sont interdits. " § 2, al. 1 du GWB reprend mot pour mot les quatre conditions d'exemption de l'article $81, \S 3$ du Traité CE. L'abandon du système du contrôle de l'abus des restrictions verticales en faveur du système d'interdiction a été sévèrement critiqué par certains auteurs comme " un pas en arrière vers le XIX siècle » (Wernhard Möschel).

L'alignement du droit allemand sur le droit communautaire a été toutefois favorisé par l'assouplissement du droit de la concurrence communautaire en matière des restrictions verticales. En effet, la Commission s'était engagée depuis 1996 dans une action qualifiée de modernisation de la politique de concurrence, afin de l'adapter aux réalités du monde économique contemporain. Grâce aux conclusions des travaux des économistes, la Commission s'est rendu compte qu'en présence d'une pression inter-marques, la structure verticale est amenée à transférer ses bénéfices vers les consommateurs. Les effets négatifs des ententes verticales ne sont susceptibles d'apparaître que lorsque le fabricant dispose d'un pouvoir de marché assez important pour ne pas craindre la concurrence d'autres marques. C'est dans ce sens que les règlements actuels dits de " nouvelle génération » (tels que le Règlement (CE) $n^{\circ}$ 2790/1999 concernant les accords verticaux, mais aussi le Règlement (CE) $n^{\circ} 1400 / 2002$ concernant le secteur automobile ou encore le Règlement (CE) $n^{\circ} 772 / 2004$ concernant les accords de transfert de technologies) font dépendre la présomption de licéité du pouvoir de marché de l'entreprise (en principe jusqu'à $30 \%$ de parts de marché). II s'agit là d'une approche plus soucieuse des effets des accords sur le marché.

Le droit allemand a non seulement adhéré au principe d'interdiction assortie de l'exemption, mais prévoit également l'application analogue des règlements d'exemption par catégorie communautaire, même s'il s'agit d'une affaire purement allemande ( $\$ 2$, al. 2 du GWB). Le droit national renvoie donc aux règlements européens qui s'appliquent également en droit allemand interne. Le mécanisme de ce renvoi est original : il s'agit d'un « renvoi dynamique » (dynamische Verweisung) qui présente l'avantage, à la différence du " renvoi statique » (statische Verweisung), qu'en cas de modification d'un règlement d'exemption communautaire, une modification de la loi allemande n'est pas nécessaire, car les changements seront automatiquement intégrés en droit allemand. Même si le législateur allemand abandonne ainsi une partie de sa souveraineté, ce système présente l'avantage, du point de vue des entreprises, que droit allemand et droit communautaire restent continuellement identiques en la matière. Les acteurs économiques n'ont ainsi pas à tenir compte d'une législation nationale différente lors de la constitution de leurs réseaux de distribution en Europe.

Ce level playing field se répand aussi en France, non pas en raison de l'intervention du législateur, mais grâce à la pratique décisionnelle. En effet, l'objectif traditionnel que poursuit le Conseil de la concurrence français est de "ne pas introduire de discordance entre les jurisprudences nationales et communautaires ". En la matière, le Conseil de la concurrence a reconnu une place importante au Règlement communautaire au sein même de l'ordre juridique interne, en déclarant que le Règlement (CE) $n^{\circ} 2790 / 1999$ peut " constituer un guide d'analyse pour la mise en œuvre du droit national » (Conseil de la concurrence, décision n01-D-45, du 19 juillet 2001, relative à une saisine présentée par la société Casino France)

Si le droit communautaire apparaît comme une source d'inspiration importante de la pratique décisionnelle des autorités et juridictions françaises, le dispositif français reste cependant moins fidèle aux règles communautaires que le droit allemand, qui procède par renvoi littéral aux textes communautaires.

\section{Les implications pratiques pour les entreprises}

L'engagement au respect d'un prix fixe ou minimum lors de la revente est prohibé
Le droit allemand interdit les clauses entre le fabricant et le distributeur indépendant par lesquelles ce dernier s'engage à respecter un certain prix fixe ou prix minimum au moment de la revente, exception faite dans le domaine de l'édition en raison de considérations politico-culturelles (cf. la Loi sur le prix unique du livre: Gesetz über die Preisbindung für Bücher). Ces clauses sont jugées particulière- 
ment nocives puisque l'autonomie des revendeurs dans la fixation de leurs prix est considérée comme l'essence même de leur indépendance commerciale. En présence d'une clause de prix fixe ou minimum, tout l'accord est exclu du champ d'application des Règlements d'exemption. Cette conséquence d'importance est traduite par la notion de « clause noire ». Etant donné qu'il est très improbable qu'une telle clause puisse bénéficier d'une exemption individuelle, les prix fixes et minima sont, en principe, condamnés par le droit allemand en application du $\S 1$ du GWB. Le constat est le même si l'affaire affecte le commerce entre États membres de l'Union européenne par application de l'article 81, § 1 du Traité CE.

\begin{abstract}
Aux Etats-Unis, pendant longtemps, les prix fixes et minima étaient également frappés d'une illicéité automatique en vertu de l'article $1^{\mathrm{er}}$ du Sherman Act. En 2007, la Cour suprême est revenue sur cette jurisprudence quasi centenaire, à l'occasion de son arrêt Leegin Creative Leather Products, Inc. v. PSKS, Inc. (127 S.Ct. 2705) : un fabricant de produits en cuir, Leegin, avait suspendu les livraisons à des distributeurs qui revendaient en deçà des prix conseillés. Les juges ont fait échapper cette pratique à l'interdiction.

La sévérité allemande et communautaire à l'égard des prix fixes et minima est discutable et, à la lumière du récent revirement américain, de plus en plus discutée. Certes, de telles clauses éliminent la concurrence entre distributeurs, mais au seul plan de la concurrence intra-marque par les prix. En revanche, des études économiques ont révélé que l'imposition des prix peut avoir des effets bénéfiques. Les prix imposés sont un moyen efficace de lutte contre la « double marginalisation » : un producteur qui se trouve en situation de monopole sur un marché vend son produit au distributeur à un prix supérieur au coût marginal. Le distributeur intègre ce prix et, s'il se trouve également en situation de monopole, revend à son tour le produit à un prix supérieur au prix marginal. Si le fournisseur et le distributeur calculent alors leurs marges de profit de manière autonome, le cumul de ces marges aboutira à un surprofit important et à des prix élevés. Cela nuit à l'efficacité du réseau. En revanche, par le biais des prix imposés, le prix de revente peut être fixé afin de maximiser le profit de la structure de distribution dans son ensemble. En outre, les prix imposés permettent de maintenir l'image de marque du réseau et de garantir la qualité de ses services.
\end{abstract}

L'Allemagne a fait pendant longtemps figure d'exception en matière des prix maxima ; mais ici, à l'inverse de son attitude générale, elle s'est montrée particulièrement sévère. L'ancien $\S 14$ du GWB prohibait toute entente entre entreprises limitant la liberté d'une des parties de fixer ses prix de revente. L'interdiction s'étendait à toutes les formes d'imposition de prix, y compris aux prix maxima. En matière de prix conseillés, l'Allemagne se distinguait également très nettement des autres ordres juridiques, puisqu'ils y faisaient l'objet d'une interdiction de principe, toutefois atténuée par des exceptions légales dont la plus importante était celle autorisant la recommandation d'un prix non-obligatoire pour des produits de marque (unverbindliche Preisempfehlung für Markenwaren), prévue par l'ancien $\S 23$, al. 1 du GWB. Depuis la dernière réforme, le fabricant peut imposer un prix de revente maximum (en effet, la fixation d'un montant maximum au-dessus duquel il ne faut pas faire payer les clients peut être facilement identifiée comme étant conforme à l'intérêt du consommateur sans devoir pousser beaucoup plus avant l'analyse) ou conseiller un prix, à la condition que ces pratiques n'équivalent pas à un prix fixe ou minimum en raison de pressions exercées par le fabricant pour le cas d'un non respect du prix maximum ou conseillé.

Un fabricant peut désirer organiser son réseau en attribuant différents territoires à ses distributeurs. Cela lui permet notamment d'exiger que ces derniers fournissent des services tels que le conseil, ou le service après-vente qu'ils ne pourraient se permettre de proposer s'ils étaient soumis à la concurrence d'autres vendeurs de la même marque. Les clients chercheraient en effet à bénéficier des conseils et services du distributeur tout en achetant le produit auprès d'autres vendeurs, appelés free-riders, qui vendraient moins chers car ils n'assumeraient pas les frais de ces services. Le fournisseur peut considérer ces services comme nécessaires à l'organisation d'un réseau face aux autres marques. Mais pour que chaque revendeur bénéficie d'une exclusivité sur un territoire, il faut que les autres revendeurs respectent son exclusivité et se voient, de ce fait, imposer des restrictions. A titre d'exemple, le distributeur ne sera pas autorisé à vendre les produits ou les services hors de sa zone d'exclusivité, ou alors il pourra être obligé de travailler seulement à partir d'un lieu précis.
En revanche, le conseil d'un prix ou l'imposition d'un montant maximum sont des pratiques licites

Restrictions territoriales / exclusivité territoriale ... 
... clauses prohibées à quelques exceptions près
D'autres clauses ne sont licites que sous certaines conditions

La légalité de la clause de non-concurrence dépend de sa durée : 5 ans pendant le contrat ...
En raison de cet effet particulier consistant à répartir les marchés entre distributeurs, le droit de la concurrence interdit les clauses d'exclusivité absolue. II s'agit ici de nouveau d'une clause dite « noire » qui fait tomber le contrat tout entier. Quelques exceptions sont toutefois prévues, dont la plus importante recouvre la possibilité d'interdire la pratique des ventes actives (c'est-à-dire les ventes qui résultent de la prospection de clients de la part du distributeur). Toutefois, les ventes passives (c'est-à-dire les ventes que le distributeur n'a pas provoquées) doivent toujours rester possibles pour le commerçant indépendant.

Le principe d'interdiction des restrictions territoriales connaît plus précisément quatre exceptions qui, pour la plupart, sont nécessaires à la mise en place d'un système de distribution exclusive ou sélective. Sont ainsi admises :

- (1) en présence d'une exclusivité de distribution, les restrictions imposées aux autres distributeurs à condition que seules les ventes actives soient visées. Le distributeur doit toutefois être libre de pratiquer des ventes passives:

- (2) la restriction des ventes aux utilisateurs finaux par un acheteur qui opère en tant que grossiste sur le marché ;

- (3) les clauses qui interdisent aux membres d'un réseau de distribution sélective de revendre à un membre non agréé. En effet, dans le cadre d'un réseau de distribution sélective, le fournisseur interdit de vendre ses produits à des distributeurs qu'il n'a pas choisis. La restriction des ventes par les membres du réseau aux distributeurs non agréés est permise, car elle est nécessaire à l'existence et au fonctionnement du réseau de distribution sélective ;

- (4) et, finalement, la restriction de la capacité du revendeur de vendre des composants destinés à l'incorporation à des clients qui pourraient utiliser ces composants pour la fabrication de biens similaires à ceux produits par le fournisseur des composants.

Concernant la vente en ligne : en général, le recours à Internet n'est pas considéré comme une forme de vente active car il s'agit d'un moyen raisonnable pour atteindre la clientèle. Un fournisseur ne peut donc pas imposer aux distributeurs une interdiction catégorique de vente via leurs sites Internet. En revanche, un message non sollicité, transmis par courrier électronique à des clients individuels ou à un groupe de clientèle déterminé, est considéré comme une vente active et peut, de ce fait, être interdite.

Certaines clauses qui sont bien souvent nécessaires à l'établissement et au fonctionnement du réseau de distribution présentent toutefois également un risque d'atteinte à la concurrence. Tel est le cas de la clause de non-concurrence, définie comme " toute obligation directe ou indirecte interdisant à l'acheteur de fabriquer, d'acheter, de vendre ou de revendre des biens ou des services qui sont en concurrence avec les biens ou les services contractuels » (article 1er lit. b du Règlement $\left.n^{\circ} 2790 / 1999\right)$. Pour mieux comprendre l'ambivalence concurrentielle de cette clause, on pourra l'illustrer avec l'exemple d'un réseau de franchise: l'essentiel de la valeur d'une franchise réside dans le savoir-faire que transmet le franchiseur à ses franchisés. Si cette mise à disposition est nécessaire à l'établissement d'un réseau de franchise, le franchiseur court le risque que son savoirfaire soit exploité par ses concurrents. Les clauses de non-concurrence permettent au franchiseur d'établir son réseau, tout en protégeant son savoir-faire et sont, de ce point de vue, favorables à la concurrence. Toutefois, en empêchant le franchisé, ou ancien franchisé qui a désormais une bonne connaissance du marché, de le concurrencer, ces clauses sont également susceptibles de porter atteinte à la concurrence.

Afin de surmonter ce conflit, le droit de la concurrence prévoit un mécanisme d'autorisation restreinte : il est interdit d'imposer une obligation de non-concurrence dont la durée est indéterminée ou dépasse cinq ans (article 5 lit. a du Règlement $\left.n^{\circ} 2790 / 1999\right)$. En revanche, si l'engagement de non-concurrence est de cinq ans ou moins et si le fournisseur ne dispose pas de plus de $30 \%$ de parts de marché, la clause bénéficie de l'exception légale dudit Règlement. La limite des cinq ans ne joue pas lorsque le distributeur exerce dans des locaux dont le fournisseur est propriétaire ou lorsque le fournisseur loue à des tiers non liés au revendeur. La durée de la clause de non-concurrence peut alors être plus longue, mais ne doit pas dépasser la période d'occupation des locaux. En outre, 
certaines spécificités seront prises en compte par la pratique décisionnelle, telles que le transfert d'un savoir-faire substantiel ou le maintien de l'identité commune et de la réputation du réseau qui peuvent justifier le plus souvent une clause de non-concurrence pendant toute la durée du contrat.

Concernant les clauses de non-concurrence post-contractuelles, celles-ci empêchent le distributeur, après l'expiration du contrat, d'exercer directement la même activité, ou d'intégrer un réseau susceptible de concurrencer le fournisseur. Sous l'ancien système allemand, les clauses de non-concurrence postcontractuelles étaient généralement justifiées si elles s'avéraient indispensables au fonctionnement du réseau. Elles devaient être proportionnées à ce but, c'està-dire limitées dans la durée, dans l'espace et quant à leur objet. Suite à la réforme de 2005, une clause de non-concurrence post-contractuelle n'est plus légale sauf si certaines conditions cumulatives sont remplies : (1) l'obligation doit concerner des biens ou des services qui sont en concurrence avec les biens ou services contractuels ; (2) elle doit être limitée aux locaux et aux terrains à partir desquels l'acheteur a opéré pendant la durée du contrat ; (3) elle doit être indispensable à la protection d'un savoir-faire transféré par le fournisseur à l'acheteur ; (4) sa durée doit être limitée à un an à compter de l'expiration de l'accord (article 5 lit. b du Règlement $\left.n^{\circ} 2790 / 1999\right)$ et (5) la part de marché du fournisseur ne doit pas excéder $30 \%$ (article 3 du Règlement $n^{\circ}$ 2790/1999).

La limitation aux locaux rend cette exception sans intérêt en pratique. Le distributeur pourra exercer une activité concurrentielle juste à quelques mètres de son ancien emplacement et capter ainsi la clientèle. En outre, le terme " indispensable » à la protection d'un savoir-faire qui se substitue à celui de « nécessaire » anciennement exigé, rend la preuve difficile à apporter.

La clause d'approvisionnement exclusif peut se révéler nécessaire au bon fonctionnement d'une structure verticale. Elle permet en effet au fournisseur de s'assurer de l'homogénéité et de la qualité des produits vendus ou utilisés par ses distributeurs. Prenons l'exemple d'un entrepreneur qui veut se lancer, par le biais d'un système de franchise, sur le marché de la restauration autour d'un concept de gastronomie bovine. Si sa stratégie commerciale se fonde sur une sélection rigoureuse de viande haut de gamme qu'il met en avant dans ses publicités, le succès de l'implantation du réseau dépendra du suivi uniforme de cette stratégie par les franchisés. II suffirait qu'un certain nombre de franchiseurs ne conforment pas leurs achats de matière première à l'image de qualité communiquée au public pour empêcher la reconnaissance du réseau par le consommateur. La clause d'approvisionnement exclusif, soit auprès du franchiseur lui-même, soit auprès d'une liste de fournisseurs homologués et contrôlés régulièrement par le franchiseur, permettra de veiller à la qualité homogène de la viande vendue par le réseau. Cependant, ces clauses ont également un effet négatif car elles ferment l'accès au marché à d'autres fournisseurs.

Le $\S 1$ du GWB permet de sanctionner les effets anticoncurrentiels des clauses d'approvisionnement exclusif. L'interdiction n'est toutefois pas absolue. Le Règlement $n^{\circ} 2790 / 1999$ assimile en son art. $1^{\text {er }}$, lit. b l'obligation d'approvisionnement exclusif ou quasi-exclusif (si sont visés plus de $80 \%$ des achats annuels) à l'obligation de non-concurrence pendant la durée du contrat. Une telle clause bénéficie donc de l'exemption du Règlement si sa durée ne dépasse pas cinq ans et si le pouvoir de marché du franchiseur n'excède pas les $30 \%$. En outre, une telle clause peut être considérée licite si elle est nécessaire au maintien de l'identité commune et de la réputation du réseau et que sa durée ne dépasse pas celle du contrat.

LES ÉVOLUTIONS SUCCESSIVES DU MARCHÉ ET DE LA SOCIÉTÉ s'accélèrent, et il incombe au droit de la concurrence de discerner les régimes favorables à ces étapes et ceux qui leur sont nuisibles. Cette tâche est difficile puisqu'il n'y a pas qu'une seule réponse valable. Pour discriminer entre les différentes solutions possibles, le législateur s'appuie sur les idées politiques et économiques dominantes. Ces idées étant évolutives, le droit de la concurrence varie selon
... et un an après

l'expiration de celui-ci

La légalité de la clause d'approvisionnement exclusif...
... dépend également d'une durée relative ou d'une nécessité spécifique 
l'époque dans laquelle il s'inscrit et suivant les nécessités auxquelles il doit faire face. Ainsi, de grandes modifications jalonnent le droit de la concurrence, et celles intervenues ces dernières années en Allemagne s'expliquent notamment par le contexte spécifique de l'intégration du marché communautaire. Ce mouvement continue et atteindra un nouveau sommet en 2010, année où les règlements communautaires expireront.

Contrairement à la France, où l'alignement au droit européen repose sur l'idée du respect de l'esprit des règles européennes davantage que sur leur application à la lettre, toute modification des règlements communautaires amènera automatiquement le même changement en droit allemand. II reste ainsi à espérer que l'évolution du droit européen continuera dans la direction de modernisation choisie. En effet, si des règles juridiques sont nécessaires afin d'éviter des aberrations ou des excès, le législateur doit également laisser aux acteurs économiques la possibilité de faire face aux nécessités de l'organisation de la distribution en réseau, les accords de distribution étant surtout destinés à favoriser la création d'un tissu de rapports économiques couronné de succès sur le marché.

\section{Indications bibliographiques :}

-BAHR C., "Die Behandlung von Vertikalvereinbarungen nach der 7. GWB-Novelle », Wirtschaft und Wettbewerb, $\mathrm{n}^{\circ}$ 3, 2004, p. 259-266

-BLAISE J.-B., IDOT L., Chronique «Concurrence », Revue trimestrielle de droit européen, $\mathrm{n}^{\circ} 2$, 2008, p. 313-403

- COMMISSION EUROPÉENNE, Rapport sur les opportunités et les freins du commerce électronique en Europe (http://ec.europa.eu/competition/consultations/2009_online_commerce/roundtable_report_en.pdf)

- Ferrier D., Droit de la distribution, $5^{\mathrm{e}}$ éd., Paris, Litec, 2008

- Kahlenberg H., Haellmigk C., « Neues Deutsches Kartellgesetz. Stichtag 1.7.2005 : Änderung des GWB », Betriebsberater, n 28/29 2005, p. 1509-1516

- Philippe J., Idot L., Gyselen L., Jenny F., Sevy D., Parplies K., Kallaugher J. "Anticompetitive foreclosure and the coherence of competition policy towards vertical restraints ", Concurrences, $n^{\circ} 42008, n^{\circ} 22113$, www.concurrences.com

- Philippe J., Nasse P., Peeperkorn L., Fox E., Perrot A., « The law and economics of resale price maintenance: A comparative perspective ", Concurrences, $n^{\circ} 42008, n^{\circ} 22111$, www. concurrences.com

- Vogel J., "Vers un retour de la distribution automobile au droit commun des restrictions verticales ? ", Concurrences, $n^{\circ}$ 4, 2008, p. 64-69

- Vogel L., Droit des ententes et des abus de domination : 360 décisions commentées, Paris, Lawlex, 2008. 